\title{
Executive Function and its Relevant Factors in Patients with Major Depressive Disorders
}

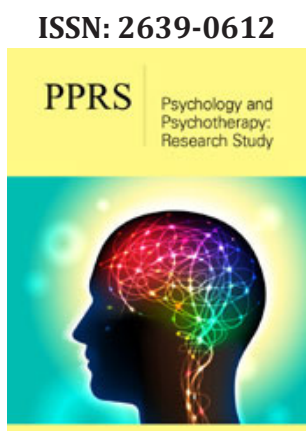

*Corresponding author: Yongmei $\mathrm{H}$, Department of Psychology, School of Humanities and Management, Guangdong Medical University, Guangdong Province, China

Submission: 㘹April 23, 2021

Published: 觜 May 06, 2021

Volume 4 - Issue 5

How to cite this article: Yongmei $\mathrm{H}$, Kexin T. Executive Function and its Relevant Factors in Patients with Major Depressive Disorders. Psychol Psychother Res Stud. 4(5). PPRS. 000599. 2021. 2021. DOI: 10.31031/PPRS.2021.04.000599

Copyright@ Yongmei H, This article is distributed under the terms of the Creative Commons Attribution 4.0 International License, which permits unrestricted use and redistribution provided that the original author and source are credited.

\section{Yongmei $\mathrm{H}^{*}$ and Kexin T}

Department of Psychology, School of Humanities and Management, Guangdong Medical University, Guangdong Province, China

\author{
Abstract \\ Objective: To explore the characteristics of executive function and its relevant factors among Patients \\ with Major Depressive Disorders (MDD).
}

Methods: Two hundred and sixty-five patients with major depressive disorders were selected, who were admitted to the $4^{\text {th }}$ neurological department in Guangdong Sanjiu Brain Hospital from July 2018 to June 2020. They were administed with Hamilton Depression Rating Scale (HAMD), Hamilton Anxiety Rating Scale (HAMA), Wisconsin Card Sorting Test (WCST) and a self-edited questionnaire on the personal general information.

Result: The total score of HAMA, HAMD and WCST were $(16.32 \pm 5.91)$, (34.67 \pm 8.72$)$ and $(1.97 \pm 1.94)$, respectively. Multiple linear regression analysis showed that the following 7 factors such as education, family monthly income, taking antidepressant drugs as prescribed, length of exercise per day, sleep quality, regular physical therapy, as well as understanding and support from relatives and friends were postively related with the total score of WCST $(B=.137 \sim .522$, all $\mathrm{P}<.05)$, and the total score of HAMD and HAMA, age, age of first episode, duration of depression, recurrent depression and family history of psychosis were negatively related with the total score of WCST $(\mathrm{B}=-.215 \sim-.711$, all $\mathrm{P}<.05)$.

Conclusion: The MDD patients' executive function has decreased significantly, which is closely related to such factors as the course of depression, severity of depression, the degree of brain function decline, treatment compliance, as well as family and social support.

Keywords: Major depressive disorder; Executive function; Relevant factors

Abbreviations: MDD: Major Depressive Disorder; HAMD: Hamilton Depression Rating Scale; HAMA: Hamilton Anxiety Rating Scale; WCST: Wisconsin Card Sorting Test

\section{Introduction}

Major Depressive Disorder (MDD) is a common mental disease, which is characterized by low mood, slow thinking, weak willpower, a variety of physical symptoms, cognitive impairment and so on [1]. According to the WHO (WHO) report, the incidence of MDD in the world is $4.4 \%$, and the incidence among female and male is $5.1 \%$ and $3.6 \%$, respectively [2]. The lifetime prevalence of MDD in China is 3.4\% [3]. MDD has the characteristics of high prevalence, low cure rate, high recurrence rate, high disability rate and high suicide rate, ranking the fourth among the world's disability diseases, and will become the first disease burden in the world by 2030 [2]. A large number of studies [4-7] have pointed out that there are comprehensive and persistent cognitive impairment in MDD patients after the first episode, including the impairment of orientation, memory, attention, learning ability, calculation, analysis, judgment, executive function and other cognitive functions, especially the executive function impairment reflecting frontal lobe function, and the impairment of cognitive function in elderly patients is more severe than that in young patients. Severe depression patients not only have cognitive impairment in the acute stage, but also have some cognitive impairment in the remission stage, which seriously affects patients' social life, family life, study and work, and increases the risk of depression recurrence [8], and the risk of Alzheimer's disease [9].

Executive function refers to the ability of an individual to make comprehensive use of all kinds of resources around him to solve problems in an unfamiliar environment. It is generally considered to include three basic parts: working memory, reaction inhibition and the ability to switch between tasks [10]. The executive dysfunction of MDD patients $[10,11]$ is mainly reflected in the impairment of cognitive flexibility, conversion and reaction inhibition, the decline of decision-making and task management ability, and the lack of self-confidence. Functional imaging studies have shown that the executive dysfunction of patients with MDD 
may be related to the structural abnormalities of the prefrontal lobe and the subcutaneous nuclei connected with the prefrontal lobe, and the dysfunction of the prefrontal lobe [11]. At present, the research on executive function of patients with MDD is mainly based on epidemiological investigation, and the conclusions of various studies are not consistent. Some studies have found that patients with MDD have significant defects in several areas of executive function, but other studies have found that there is no significant difference in executive function between patients with MDD and healthy people [12]. Therefore, the executive function of patients with MDD is likely to be affected by some factors. However, there are few studies on the influencing factors of executive function in patients with MDD. This study aims to explore this problem.

\section{Object and Methods}

\section{Object}

Sampling: 265 patients with MDD were selected from July 2018 to June 2020 in the fourth neurologic Department of Guangdong Sanjiu brain hospital. Among them, there are 153 men and 112 females; with the average age of $(31.5 \pm 14.8)$ years old; average duration of disease of $(43.4 \pm 56.2)$ month, first-episode age of (28.3 \pm 13.9$)$ years old. Inclusion criteria: (1)Han nationality; (2)1260 years old; (3) meet the diagnostic standard of depression in ICD-10, the 10th edition of international classification of diseases. (4) diagnosed as depression by psychiatrist; (5) total score of Hamilton Depression Scale (HAMD) of 24 items $\geq 20$. Exclusion criteria: (1) with chronic, serious physical disease, or severe mental illness attack; 20 alcohol and drug dependence history; (3) color blindness; (4) brain organic diseases; (5) pregnant and lactating women.

Informed consent: The enrolled patients were explained the purpose, procedure and precautions of the study in detail and asked to sign informed consent.

Ethical review: The procedure and content of this study are in accordance with the ethical standards set by the ethics committee of Guangdong Sanjiu Brain Hospital and have been approved by the Committee.

\section{Tools}

Hamilton Depression Rating Scale (HAMD): It is compiled by Hamilton [13] and revised by Tang Yuhua to Chinese version [14]. There are 24 items, divided into 7 factors: anxiety/ somatization, weight, cognitive impairment, day and night changes, delay, sleep disorders, despair. The liketer 5-points scoring method is used to score from 0 to 4 points corresponding to (asymptomatic) - (very heavy). The higher the score, the more serious the depression. The clinical demarcation points are: If the total score $<8$, the patient is considered normal; If the total score ranges 8 to 19 , the patient is possible depression; If the total score ranges 20 to 35 , the patient is positive depression; and if the total score $>35$, the patient is severe depression. In this study, the cronbach'a coefficient of the total scale is 0.84 , and the cronbach'a coefficient of each factor is $0.74-0.79$.

Hamilton Anxiety Rating Scale (HAMD): It is compiled by Hamilton [15], revised by Tang Yuhua to Chinese version [16].
There are 14 items, which are divided into two factors: physical anxiety and mental anxiety. The higher the score, the more anxious the anxiety. The liketer 5-points scoring method is used to score from 0 to 4 points corresponding to (asymptomatic) - (very heavy). The clinical demarcation points are: If the total score $<7$, the patient is considered normal; If the total score is 7 to 13 , the patient is possible anxiety; If the total score is $14-20$, the patient is positive anxiety; If the total score is $21-28$, the patient is obvious anxiety; And if the total score is more than 28, the patient is severe anxiety. In this study, Cronbach 'a coefficient of the total scale is 0.90 , and Cronbach' a coefficient of each factor is 0.79-0.86, respectively.

Wisconsin Card Sorting Test (WCST): It is compiled by Grant (1948) and revised by Tan Yunlong to Chinese version [17], which is used to test the abstract thinking ability of non-color blind people over 6 years old. There are 4 stimulus cards and 128 response cards, which are divided into 14 test indexes, namely Total Score (TS), number of Complete Categories (CC), Percentage of Conceptualization Level (RFP), Continuous Responses (RP), Continuous Error (RPE), non-continuous error (NRPE), total responses required to complete the test (RA), Correct Responses (RC), Correct Response Percentage (RCP), Wrong Responses (RE), the number of answers required to complete the first classification (RF), the Percentage Of Persistent Errors (RPEP), the number of incomplete classifications (FM), and the number of Learning to Learn (LL). In this study, the cronbach'a coefficient of the total scale is 0.91 , and the cronbach'a coefficient of each dimension is 0.75 0.86 .

Self-compiled questionnaire on personal general information: The CNKI, Wanfang database, VIP database, Baidu, Pubmed and other search engines are used to search the literatures about "related factors of executive function among major depressive disorder" (176 in Chinese and 4733 in foreign). Based on that, the basic content of the questionnaire is constructed, with a total of 17 items. Combined with the results of 3 collective discussions with 9 representatives of psychiatrists, 1 item is deleted, and 2 items are added. The final questionnaire for personal general information of MDD patients consists of 18 items, including age, gender, age of first episode, depression duration (months), whether anxiety is combined, education, occupation, whether psychological treatment has been received, monthly income of family, whether taking antidepressants according to the doctor's orders, whether exercise for more than 30 minutes every day, sleep quality, whether recurrence depression, with family history of mental diseases or not, with major negative life events within last 1 year or not, whether physical therapy (such as trams) was regularly accepted, what the attitude of relatives and friends towards your depression, and depression subtypes, etc.

\section{Data processing}

SPSS 20.0 is used to analyze the effective data. Descriptive statistics are used to calculate the average score and standard deviation of each scale; Pearson product difference correlation, independent sample t-test and one-way ANOVA are used to explore the pairwise correlation among the variables; multiple linear regression is used to analyze the main related factors of WCST total score. 
Results

Descriptive statistics of total score and factor score of each scale

It can be seen from Table 1 that the HAMD total score of this group reached the standard of (positive) depressive symptoms [9], HAMA total score reached the standard of (positive) anxiety symptoms [10], and WCST total score was significantly lower than that of normal people [17].

\section{Correlation analysis of scores of each scale}

From Table 2, there was a significant positive correlation between total scores of HAMD , HAMA and WCST.

Table 1: Descriptive analysis of total score and each factor score of 3 scales $(n=265)$.

\begin{tabular}{|c|c|c|c|c|}
\hline Factor & M & SD & Min & Max \\
\hline Total score of HAMD & 34.67 & 8.72 & 21 & 50 \\
\hline Anxiety or somatization & 9.02 & 2.18 & 4 & 13 \\
\hline Weight & 0.61 & 0.63 & 0 & 2 \\
\hline Cognitive impairment & 7.36 & 3.49 & 2 & 17 \\
\hline Diurnal variation & 0.55 & 0.61 & 0 & 2.01 \\
\hline Delay & 6.25 & 2.03 & 1 & 13 \\
\hline Sleep disorders & 5.43 & 1.88 & 2 & 8 \\
\hline Despair & 5.45 & 2.19 & 2 & 10 \\
\hline Total score of HAMA & 16.32 & 5.91 & 5 & 29 \\
\hline Mental anxiety & 10.81 & 3.51 & 4 & 19 \\
\hline Somatic anxiety & 5.53 & 3.01 & 0 & 12 \\
\hline Total score of WCST & 1.97 & 1.94 & 0 & 6 \\
\hline Number of categories completed & 2.01 & 1.73 & 0 & 6 \\
\hline Percentage of conceptualization level & 30.76 & 21.88 & 0 & 85.71 \\
\hline Continuous response & 40.86 & 24.35 & 2 & 109 \\
\hline Persistent error & 16.97 & 15.01 & 0 & 60 \\
\hline Non persistent error & 47.62 & 28.97 & 6 & 103 \\
\hline Total number of answers to complete the test & 103.35 & 10.79 & 70 & 128 \\
\hline Correct answers & 52.79 & 18.03 & 25 & 93 \\
\hline Correct response percentage & 46.99 & 17.27 & 19.53 & 88.57 \\
\hline Wrong answers & 66.61 & 23.44 & 8 & 103 \\
\hline Number of answers required to complete the first category & 54.87 & 29.98 & 10 & 128 \\
\hline Percentage of persistent errors & 13.89 & 11.85 & 0 & 47.62 \\
\hline Unable to maintain the full number of categories & 1.35 & 1.8 & 0 & 8 \\
\hline Learning to learn & 0.08 & 0.14 & -0.15 & 1.1 \\
\hline
\end{tabular}

Table 2: Correlation analysis of total score of HAMD, HAMA and WCST ( $\mathrm{n}=265)$.

\begin{tabular}{|c|c|c|c|}
\hline Factor & Total Score of HAMD & Total Score of HAMA & Total Score of WCST \\
\hline 1. Total score of HAMD & & & \\
\hline 2. Total score of HAMA & $.827^{* *}$ & & \\
\hline 3. Total score of WCST & $-.218^{* *}$ & $-.106^{* *}$ & \\
\hline
\end{tabular}

Note: ${ }^{* *} \mathrm{P}<0.01$.

Univariate analysis of demographic variables of (alternative answers) of demographic classification variables and executive function among MDD patients

Variable assignment: Firstly, the possible situations clinical related classification variables that may affect the total score of WCST are assigned. The results are shown in Table 3. 
Table 3: SVariable assignment.

\begin{tabular}{|c|c|c|}
\hline S. No. & Item & Options and Assignment \\
\hline 1 & Gender & $0=$ male, $1=$ female \\
\hline 2 & education & $\begin{array}{c}0=\text { illiterate, } 1=\text { primary school, } 2=\text { junior high school, } 3=\text { senior high } \\
\text { school or technical secondary school, } 4=\text { junior college, } 5=\text { Undergraduate } \\
\text { or above }\end{array}$ \\
\hline 3 & Occupation & $\begin{aligned} 0=\text { civil servant, } 1 & =\text { worker, } 2=\text { farmer, } 3=\text { businessman, } 4=\text { manager, } 5= \\
\text { teacher, } 6 & =\text { medical worker, } 7=\mathrm{IT} \text { industry, } 8=\text { freelance }\end{aligned}$ \\
\hline 4 & Have you ever received regularly psychotherapy? & $0=$ none, $1=$ yes \\
\hline 5 & The family monthly income is & $\begin{array}{c}0=0 \sim 4000 \text { yuan, } 1=5001 \sim 8000 \text { yuan, } 2=8001 \sim 12000 \text { yuan, } 3=12001 \\
\text { yuan or more }\end{array}$ \\
\hline 6 & Whether taking antidepressants as prescribed? & $\begin{array}{c}0=\text { none at all, } 1=\text { not very good at taking antidepressants as prescribe } \\
\qquad 2=\text { basically take antidepressants as prescribe }\end{array}$ \\
\hline 7 & Whether anxiety is combined? & $0=$ no, $1=$ yes \\
\hline 8 & Do you exercise more than 30 minutes every day? & $0=$ no, $1=$ yes \\
\hline 9 & Sleep quality: & $0=$ very bad, $1=$ not very good, $2=$ average, $3=$ good, $4=$ very good \\
\hline 10 & Recurrent depression & $0=$ no, $1=$ yes \\
\hline 11 & Family history of mental illness & $0=$ none, $1=$ yes \\
\hline 12 & Whether there were significant negative life events in 1 year & $0=$ none, $1=$ yes \\
\hline 13 & Regular physical therapy (such as rTMS) & $0=$ no, $1=$ yes \\
\hline 14 & $\begin{array}{l}\text { What's the attitude of relatives and friends towards your } \\
\text { depression? }\end{array}$ & $\begin{array}{l}0=\text { not understanding, } 1 \text { =basically understanding but not supporting } \\
\text { treatment, } 3 \text { = basically understanding and fully supporting treatment }\end{array}$ \\
\hline 15 & Depression subtype & $\begin{array}{l}0=\text { severe depressive episode without psychotic symptoms, } 1 \text { = severe } \\
\text { depressive episode with psychotic symptoms, } 2=\text { moderate episode } \\
\text { of recurrent depressive disorder, } 3=\text { mood disorder without psychotic } \\
\text { symptoms, } 4=\text { recurrent depressive disorder with psychotic symptoms }\end{array}$ \\
\hline
\end{tabular}

Univariate analysis of executive function among MDD patients: Univariate analysis was performed on the demographic and clinical variables that may affect the total score of WCST. The results are shown in Table 4. It can be seen from Table 4 that except occupation, depression subtype, whether receive psychotherapy regularly and whether there were major negative life events within last one year, the other 14 factors had significant effects on the total score of WCST ( $r=-.305,-.248,-.346$, all $\mathrm{P}<0.001 ;|\mathrm{t}| /|\mathrm{F}|=2.121$ 9.989, all $\mathrm{P}<0.01)$.

Table 4: Univariate analysis of demographic and clinical variables that may affect the total score of WCST (n $=265$ ).

\begin{tabular}{|c|c|c|c|}
\hline S. No & Item & $\mathbf{r} / \mathbf{t} / \mathbf{F}$ & P-Value \\
\hline 1 & Age & -.305 & $<.001$ \\
\hline 2 & Gender & -4.121 & $<.000$ \\
\hline 3 & Age of first episode & -.248 & $<.000$ \\
\hline 4 & Duration of depression (months) & -.346 & $<.001$ \\
\hline 5 & Whether anxiety is combined & 3.622 & $<.001$ \\
\hline 6 & Education & -7.839 & $<.001$ \\
\hline 7 & Occupation & 1.317 & 0.274 \\
\hline 8 & Depression subtype & 2.581 & 0.054 \\
\hline 9 & Whether received psychotherapy regularly? & -1.459 & 0.417 \\
\hline 10 & Monthly family income & -3.728 & $<.001$ \\
\hline 11 & Whether taking antidepressants according to the doctor's advice & -4.376 & $<.001$ \\
\hline 12 & Do you exercise more than 30 minutes a day & -5.681 & $<.001$ \\
\hline 13 & Sleep quality & -4.471 & $<.001$ \\
\hline 14 & Recurrent depression or not & 3.947 & $<.001$ \\
\hline
\end{tabular}




\begin{tabular}{|l|c|c|c|}
\hline 15 & Family history of mental illness & 9.989 & $<.001$ \\
\hline 16 & Were There major negative life events within last 1 year? & 1.129 & 0.525 \\
\hline 17 & Do you have regular physical therapy (such as rTMS) & -7.228 & $<.001$ \\
\hline 18 & What's the attitude of relatives and friends towards your depression? & -5.213 & $<.001$ \\
\hline
\end{tabular}

Multiple stepwise linear regression analysis of executive function related factors in patients with depression

Taking the total score of WCST as the dependent variable, and the above18 demographic variables, clinical related variables and HAMA, HAMD total scores as the independent variables, the multiple stepwise linear regression analysis is carried out within 95\% confidence interval. It can be seen from Table 5 that education, family monthly income, taking antidepressants according to doctor's advice, daily exercise time, sleep quality, regular physical therapy, understanding and support from relatives and friends are positively correlated with the total score of WSCT (B=. $137 \sim$. 522 , all $\mathrm{P}<0.05$ ). The total score of WSCT is negatively correlated with HAMD, HAMA, age, first-episode age, duration of depression, recurrent depression and family history of psychosis $(B=-.215$-. 711 , all $\mathrm{P}<0.05$ ).

Table 5: Multiple stepwise linear regression analysis of main influencing factors of WSCT total score.

\begin{tabular}{|c|c|c|c|c|c|c|c|c|}
\hline \multirow{2}{*}{$\begin{array}{c}\text { Dependent } \\
\text { Variable }\end{array}$} & \multirow{2}{*}{$\begin{array}{c}\text { Independent } \\
\text { Variable }\end{array}$} & \multicolumn{2}{|c|}{ Regression Coefficient } & \multirow{2}{*}{$\beta$} & \multirow{2}{*}{$\mathbf{T}$} & \multirow{2}{*}{$\mathbf{P}$} & \multirow{2}{*}{$\mathbf{R}^{2}$} & \multirow{2}{*}{$\mathbf{R}_{\text {adj2 }}$} \\
\hline & & B & SE & & & & & \\
\hline Total score & Total score of HAMD & -0.743 & 0.054 & -0.711 & -8.148 & $<.001$ & 0.546 & 0.542 \\
\hline \multirow[t]{13}{*}{ of WCST } & Total score of HAMA & -0.646 & 0.093 & -0.608 & -6.963 & $<.001$ & & \\
\hline & age & -0.535 & 0.068 & -0.497 & -9.055 & $<.001$ & & \\
\hline & first-episode age & -0.834 & 0.121 & -0.341 & -2.559 & 0.011 & & \\
\hline & Duration of depression & -0.436 & 0.046 & -0.465 & -2.292 & 0.02 & & \\
\hline & education & 0.409 & 0.132 & 0.237 & 2.507 & 0.012 & & \\
\hline & Monthly household income & 0.472 & 0.092 & 0.352 & 2.295 & 0.02 & & \\
\hline & Taking antidepressants as prescribed & 0.384 & 0.059 & 0.433 & 2.136 & 0.023 & & \\
\hline & How long do you exercise every day & 0.313 & 0.035 & 0.251 & 3.72 & $<.001$ & & \\
\hline & Sleep quality & 0.603 & 0.079 & 0.571 & 2.691 & 0.007 & & \\
\hline & Recurrent depression or not & -0.477 & 0.068 & -0.442 & -2.888 & 0.004 & & \\
\hline & Do you have a family history of mental illness & -0.71 & 0.064 & -0.686 & -4.932 & $<.001$ & & \\
\hline & Do you receive physiotherapy regularly & 0.553 & 0.107 & 0.297 & 2.631 & 0.009 & & \\
\hline & $\begin{array}{c}\text { The attitude of relatives and friends towards your } \\
\text { depression }\end{array}$ & 0.279 & 0.083 & 0.244 & 4.102 & $<.001$ & & \\
\hline
\end{tabular}

\section{Discussion}

The total score of WCST and the score of each factor in this group are significantly lower than the normal value [15], which is consistent with the results of previous studies [16-20], suggesting that the impairment of executive function is common in MDD patients, mainly in the aspects of individual inhibition, cognitive flexibility, planning, semantic fluency and set switching ability. Multiple stepwise linear regression shows that education, family monthly income, taking antidepressants as prescribed, daily exercise time, sleep quality, regular physical therapy, understanding and support of relatives and friends are positively correlated with WCST total score; HAMD total score, HAMA total score, age, firstepisode age, duration of depression, recurrent depression and psychotic family history were negatively correlated with WCST total score.

Education and family monthly income positively predict the executive function of MDD patients, which is consistent with previous research results [21,22], suggesting that higher socioeconomic status can promote physiological and psychological functions. The "social causality theory" in the field of health [23] points out that people with lower socio-economic status (lower education level and lower per capita household consumption expenditure) are more likely to experience unfortunate life events (such as recent widowhood and death of children) Poor health level (such as malnutrition, disability, chronic diseases, infectious diseases, poor medical conditions, and so on), these factors will have a negative impact on brain function. At the same time, people with higher education level are more able to correctly understand depression and its treatment methods, more able to state their illness clearly to doctors, so that the diagnosis and treatment are more targeted, and they are more able to choose and adhere to the correct treatment methods.

Taking antidepressants according to the doctor's advice can help maintain executive function. It is consistent with the results of previous studies [21,24], suggesting that antidepressants can promote the affective disorder (such as depression) and cognitive 
function (such as executive function) of MDD patients. Based on the existing theoretical hypothesis and clinical trial results, the occurrence of depression may be influenced by many factors, and each factor plays a role through different signal pathways and pathophysiological processes, which eventually leads to changes in the number and function of synapses [25], and damages synaptic plasticity. It can reduce the excitability of cerebral cortex [26], induce the occurrence of depression, and may be accompanied by cognitive decline [27]. Therefore, the improvement of synaptic plasticity can improve depressive symptoms and cognitive function. Although classical Selective Serotonin Reuptake Inhibitors (SSRI) and new antidepressants have different mechanisms of action, their antidepressant effects are related to the improvement of synaptic plasticity [27,28]. Rational administration can promote nerve regeneration in the Dentate Gyrus (DG) and other brain regions of patients [29], so it is also conducive to the maintenance of brain function. Sleep quality positively predicts executive function in patients with depression, which is consistent with the results of previous studies $[21,29,30]$. Sleep change is the most common circadian rhythm disorder in depression, about $80 \%$ of patients with depression will have sleep disorders [30], which main clinical manifestations are difficulty in falling asleep and shortened sleep duration. Sleep is the result of the balance of brain excitation and inhibition. The poor sleep quality can reflect the inflexibility of brain excitation and inhibition, that is, the poor executive function. On the other hand, poor sleep quality hinders the recovery of various physiological functions and has extensive damage to the regulatory function of the nervous system, especially the high-level nervous function responsible for the frontal lobe, such as executive function $[31,32]$. The duration of daily exercise and regular physical therapy (such as rTMS) positively predict the executive function of patients with major depressive disorder, which is consistent with the results of previous studies [21,33-36]. It is suggested that moderate physical exercise and nerve stimulation have a positive effect on brain function. The most important clinical manifestation of depression is the decrease of mental activity, and its physiological mechanism is the decrease of synaptic number [25], function [26] and cortical excitability [26]. Experiments have shown that longterm and moderate physical exercise and regular physical therapy (such as rTMS) can increase the number of synapses [21,32-35], improve the plasticity of synapses and the excitability of cortex, thus improving executive function.

The understanding and support of relatives and friends positively predicted the executive function of patients with depression, which was consistent with previous research results [21,36], suggesting that social support can maintain the executive function of MDD patients. Patients with high degree of social support can get more material support and spiritual encouragement, which makes them more confident to adhere to treatment and get more financial resources to comply with treatment. The total score of HAMD, HAMA, age, first-episode age, duration of depression, recurrent depression and family history of psychosis were negatively correlated with the total score of WCST. It is consistent with the previous research results [21,37-42]. The above seven factors reflect the brain damage of patients and the negative impact of reduced plasticity on executive function from different perspectives.

\section{References}

1. Bayes AJ, Parker GB (2018) Comparison of guidelines for the treatment of unipolar depression: A focus on pharmacotherapy and neurostimulation. Acta Psychiatr Scand 137(6): 459-471.

2. World Health Organization (2017) Depression and other common mental disorder global health estimate. World Health organization, Switzerland, pp. 7-9.

3. Huang Y, Wang Y, Wang H (2019) Prevalence of mental disorders in China: A cross -sectional epidemiological study. Lancet Psychiatry 6(3): 211-224.

4. Clark M, DiBenedetti D, Perez V (2016) Cognitive dysfunction and work productivity in major depressive disorder. Expert Rev Pharmacoecon Outcomes Res 16(4): 455-463.

5. Albert KM, Potter GG, Mcquoid DR, Taylor WD (2018) Cognitive performance in antidepressant-free recurrent major depressive disorder. Depress Anxiety 35(8): 694-699.

6. Min Z, Zhong X, Ben C (2018) Characteristics of cognitive function and psych behavioral symptoms of senile depression during attack and recovery. Chinese Journal of neuropsychiatric diseases 8 (44): 466-470.

7. Pap Akostas GI, Culpepper L (2015) Understanding and managing Cognition in the depressed Patient. J Clin Psychiatry 76(4): 418-425.

8. Saragoussi D, Touya M, Haro JM, Jönsson B, Knapp M, et al. (2017) Factors associated with failure to achieve remission and with relapse after remission in patients with major depressive disorder in the PERFORM study. Neuropsychiatr Dis Treat 13: 2151-2165.

9. Quattropani MC, Lenzo V, Armieri V, Filastro A (2018) The origin of depression in Alzheimer disease: a systematic review. Riv Psichiatr 53(1): 18-30.

10. Asa H, Guro A (2009) Cognitive function in major depression-a summary. Front Hum Neurosci 3: 26.

11. Porter RJ, Gallagher P, Thompson JM, Young AH (2003) Neurocognitive impairment in drug-free patients with major depressive disorder. Br J Psychiatry 182(3): 214-220.

12. Grant MM, Thase ME, Sweeney JA (2001) Cognitive disturbance in outpatient depressed younger adults: Evidence of modest impairment. Biol Psychiatry 50(1): 35-43.

13. Hamilton M (1967) Development of a rating scale for primary depressive illness. Brit J Soc Clin Psychol 6(4): 278-296.

14.Xiangdong W, Xilin W, Hong Ma (1999) Handbook of mental health assessment scale. Chinese Journal of mental health, Beijing, China, 12: 220-223.

15. Hamilton M (1959) Assessment of anxiety by rating scale. Brit J Med Psychol 32(1): 50-55.

16. Xiangdong W, Xilin W, Ma Hong (1999) Handbook of mental health assessment scale. Chinese Journal of mental health, Beijing, China, 12: 253-256.

17. Yunlong T, Ying Q (2002) Stability analysis of common indicators in Wisconsin card sorting test. Chinese Journal of mental health 16(12): 831-833.

18. Yan Y, Dong Y, Jingping Z (2015) A study on cognitive function of refractory depression. Chinese Journal of clinical psychology 23 (1): 101-103.

19. Wentao C (2019) Comparison of cognitive function between patients with refractory depression and first-episode depression. Chinese modern doctor 57(1): 79-82. 
20. Li Tao, Hu Xiaoke, Chaowang F (2017) Relationship between serum GFAP, NSE, HCY levels and cognitive function in patients with first episode depression. Laboratory medicine and clinic 14(24): 3601-3604.

21. Tian Ge (2020) Analysis of clinical symptoms of depression patients with cognitive impairment. Chinese modern drug application 14(6): 97-98.

22. Gil VM, Gausset KA, Blasco SM, et al. (2018) Cognitive predictors of illness course at 12 months after first-episode of depression. Eur Neuropsychopharmacol 28(4): 529-537.

23. Dengheng S, Jiahong L, Haiyan L, et al. (2018) Influencing factors and rehabilitation treatment of cognitive impairment of depression. Rural Health Management in China 38(9): 1193-1196.

24. Shanshan G, Yanfang W (2020) Risk factors of cognitive function in patients with first-episode depression. Campus Psychology 18(4): 303 307.

25. Turnerr RJ, Lloyd DA (1999) The stress process and the social distribution of depression. Journal of Health \& Social Behavior 40(4): 513-529.

26. Boldrini M, Underwood MD, Hen R, Gorazd BR, Dwork AJ, et al. (2009) Antidepressants increase neural progenitor cells in the human hippocampus. Neuropsychopharmacology 34(11): 2376-2389.

27. Kang HJ, Voleti B, Hajszan T, Rajkowska G, Stockmeier CA, et al. (2012) Decreased expression of synapse- related genes and loss of synapses in major depressive disorder. Nat Med 18(9): 1413-1417.

28. Ledford H (2016) Psychopharmacology drugmakers target fog of depression. Nature 530(7588): 17.

29. Castren E, Hen R (2013) Neuronal plasticity and antidepressant actions. Trends Neurosci 36(5): 259-267.

30. Jiting X, Hui Y, Su Junpeng (2019) Study on cognitive impairment and its influencing factors in patients with depression. Modern Chinese Doctor 57(34): 164-168

31. Deying Y, Wenmei W, Li Hui (2019) Correlation between sleep quality and neurocognition in patients with depression. Chinese Journal of Health Psychology 27(2): 161-165.
32. Dumer JS, Dinges DF (2005) Neurocognitive consequences of sleep deprivation. Semin Neurol 25(1): 117-129.

33. Ramos AR, Tarraf W, Daviglus M, Davis S, Gallo LC, et al. (2016) Sleep duration and neurocognitive function in the Hispanic community health study/Study of Latinos. Sleep 39(10): 1843-1851.

34. Olson RL, Brush CJ, Ehmann PJ, Alderman BL (2017) A randomized trial of aerobic exercise on cognitive control in major depression. Clin Neurophysiol 128(6): 903-913.

35. Player MJ, Taylor JL, Weickert CS, Alonzo A, Sachdev SP, et al. (2014) Increase in PAS-induced neuroplasticity after a treatment course of transcranial direct current stimulation for depression. Journal of Affective Disorders 167: 140-147.

36. Guse B, Falkai P, Wobrock T (2010) Cognitive effects of high frequency repetitive transcranial magnetic stimulation: A systematic review. Journal of Neural Transmission 117(1): 105-122.

37. Power MJE, Lawlor BA, Kee F (2017) Social support mediates the relationships between extraversion, neuroticism, and cognitive function in older adults. Public Health 147: 144-152.

38. Tai Chuanzhen, Bao Jixue, Xu Xiufeng (2018) Influencing factors of cognitive function in patients with first-episode depression. Chinese Journal of mental health 32(10): 808-815.

39. Yu Haiting, Fuchun Z, Bo Qijing (2020) Analysis of cognitive function related factors of bipolar disorder and depressive disorder. Chinese Medical Journal 55(8): 917-919.

40. Rapp MA, Dahlman K, Sano M (2005) Neuropsychological differences between late -onset and recurrent geriatric major depression. Am J Psychiatry 162(4): 691-698.

41. Zuo Lina, Wang Yan, Zhang Kerang (2009) Effect of onset age on cognitive function of patients with major depressive disorder. Chinese Journal of Health Psychology 17(7): 780-782.

42. Habermann PY, Pohl J, Leplow B (2005) Attention and executive functions in remitted major depression patients. Journal of Affective Disorders 89(1-3): 125-135. 